\title{
NONLINEAR DYNAMICS AND ROBUST CONTROL OF A GYROPLANE ROTOR *
}

\author{
Yevgeny I. Somov ${ }^{*, \dagger}$ and Oleg Ye. Polyntsev ${ }^{\ddagger}$ \\ * Stability and Nonlinear Dynamics Research Center, \\ Mechanical Engineering Research Institute (IMASH), \\ the Russian Academy of Sciences (RAS) \\ 5 Dm. Ul'yanov Str. Moscow 119333 Russia \\ $\dagger$ Research Institute of Mechanical Systems Reliability \\ 244 Molodogvardeyeskaya Str. Samara 443100 Russia \\ yevgenysomov@front.ru e_somov@mail.ru \\ ¥ JSC Scientific \& Production Corporation IRKUT \\ 3 Novatorov Str. Irkutsk 664020 Russia \\ olegpolyntsev@mail.ru
}

\begin{abstract}
Mathematical models of a gyroplane rotor have been carried out. Their approximate analytical solutions have been obtained. Software allowing one to simulate and study a rotor dynamics has been created. Major physical features on the forced flexible oscillations of the rotor have been investigated. The results obtained have successfully been applied to design the A-002 gyroplane rotor. Copyright (c) 2005 IFAC
\end{abstract}

Keywords: gyroplane rotor, nonlinear dynamics, robust control

\section{INTRODUCTION}

At present due to new advanced technologies gyroplanes (GPs) are being created across the world. Therefore, in order to predict operational features of a wind-milling rotor it is of significance to advance the theory of auto-rotation. As against helicopter main rotor an auto-rotating rotor is revolved under the influence of an air stream rush. Thus, if a regime of operation of the wind-milling rotor will be changed its angular velocity will also be changed. The autogyro theory was first developed by Glauert and Lock, then mainly extended in 20-40th years of the last century in works of J.B. Weatley, F.J. Beiley, M.L. Mil', N.I. Kamov, A.P. Proskuryakov, V.G. Tabachnikov et al. Since helicopters appeared in the forties of the former century, the scope of research of main rotor dynamics had been mostly restricted by flapping, while auto-rotation study had been secondary.

* The work was supported by the RAS Presidium (Progr.19), the Division on EMMCP of RAS (Progr.19) and RFBR (Grant 04-01-96501)
Some assumptions of the analytical models of autorotation applied earlier do not allow one to investigate entirely a GP rotor. The problem posed can effectively be solved due to computers having great capacities. The purpose of the paper are modeling and research of nonlinear dynamics and robust stabilization by a gyroplane rotor, see Fig. 1. The rotor consists of two blades attached to a hub by means of teeter hinge allowing the rotor to execute flapping.

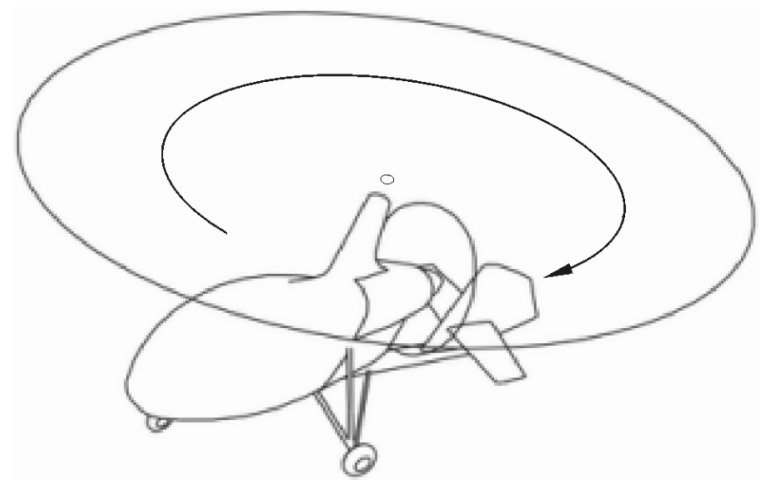

Fig. 1. The scheme of a gyroplane 


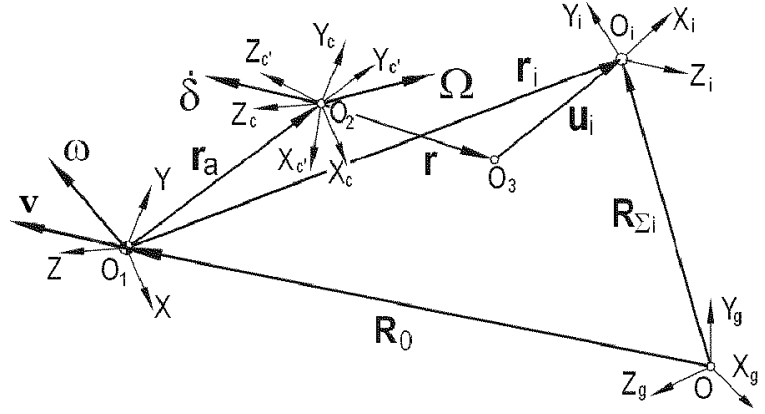

Fig. 2. The cinematic scheme of a gyroplane

\section{MODEL OF A GYROPLANE ROTOR}

Let us consider a blade element $\mathrm{O}_{\mathrm{i}}$ with distributed mass $m_{r}$, Fig. 2. The equation of motion for the element with respect to fixed reference frame is written as $m_{r} \ddot{\mathbf{r}}_{\sigma}=\mathbf{R}+\mathbf{F}$, where $\mathbf{r}_{\sigma}$ is radius-vector regarding reference mark of the Earth frame assumed to be fixed, and vectors $\mathbf{F}$ and $\mathbf{R}$ present external and internal distributed forces, respectively. The motion of flexible blades is considered. The aircraft body and a rotor-head are assumed to be absolutely rigid. Elementary forces are supposed to be applied in a mass center of the element. At the notation $\boldsymbol{\sigma}=\boldsymbol{\Omega}+\dot{\boldsymbol{\delta}}+\boldsymbol{\omega}$ an absolute acceleration vector $\ddot{\mathbf{r}}_{\sigma}$ is appeared as

$$
\begin{aligned}
\ddot{\mathbf{r}}_{\sigma}= & \dot{\mathbf{v}}+\boldsymbol{\omega} \times \mathbf{v}+\dot{\boldsymbol{\omega}} \times \mathbf{r}_{a}+\ddot{\mathbf{u}}+\dot{\boldsymbol{\sigma}} \times(\mathbf{r}+\mathbf{u}) \\
& +\boldsymbol{\omega} \times\left(\boldsymbol{\omega} \times\left(\mathbf{r}_{a}+\mathbf{r}+\mathbf{u}\right)\right) \\
& +\boldsymbol{\Omega} \times(\boldsymbol{\Omega} \times(\mathbf{r}+\mathbf{u}))+\dot{\boldsymbol{\delta}} \times(\dot{\boldsymbol{\delta}} \times(\mathbf{r}+\mathbf{u})) \\
& +2 \boldsymbol{\omega} \times((\dot{\boldsymbol{\delta}}+\boldsymbol{\Omega}) \times(\mathbf{r}+\mathbf{u}))+2 \boldsymbol{\sigma} \times \dot{\mathbf{u}} \\
& +2 \dot{\boldsymbol{\delta}} \times(\boldsymbol{\Omega} \times(\mathbf{r}+\mathbf{u}))
\end{aligned}
$$

Here $\mathbf{v}$ is vector of the GP speed; $\boldsymbol{\omega}$ is vector of angular rate by the GP body; $\mathbf{r}_{a}$ is radius-vector of the hub in respect to the GP mass center; $\boldsymbol{\Omega}$ is vector of angular rate of the blade element including auto-rotation and flapping; $\dot{\boldsymbol{\delta}}$ is vector of the control blade angular rate; $\mathbf{u}$ is vector of the blade flexible displacement.

After introducing reference frames (see Fig. 2) and transformations (1), elementary torques are defined and integrated through the lengths of the both blades from $r_{0}$ (radius of a blade root) up to $\mathrm{R}$ (radius of the rotor). As total torques regarding corresponding axes of hinges should be equal to zero, one can obtain the autorotation equation

$$
\ddot{\psi}_{\mathrm{L}} J_{\mathrm{cR}}=-M_{\mathrm{A} 1}-M_{\mathrm{A} 2}+M_{i 1}+M_{i 2},
$$

and flapping equation

$$
\ddot{\beta}_{\mathrm{L}} J_{\mathrm{pR}}=M_{\mathrm{F} 1}-M_{\mathrm{F} 2}-M_{j 1}+M_{j 2},
$$

where $\psi_{\mathrm{L}}$ is an azimuth angle of arbitrary blade; $\beta_{\mathrm{L}}$ is an angle of flapping; $J_{\mathrm{cR}}$ and $J_{\mathrm{pR}}$ are rotor's moments of inertia in respect to auto-rotation and to flapping axes, respectively; $M_{\mathrm{A} 1}, M_{\mathrm{A} 2}$ and $M_{F 1}, M_{F 2}$ are torques of internal forces causing a wind-milling and a flapping, respectively by the first and second blades; $M_{i 1}, M_{j 1}, M_{i 2}$ and $M_{j 2}$ are torques of inertia forces by both blades.

\section{PROBLEMS OF AUTOROTATION}

Describing the dynamics of the wind-milling rotor, the equations (2) and (3) are essentially nonlinear.
This fact is concerned with nonlinear dependencies of aerodynamic forces upon attack angles, Mach and Reynolds numbers, and elastic deformations; appearance of effects by flow non-stationarity; nonuniformity of mass and rigidity distribution on blade's length etc. To add, the blades are under the influence of non-symmetrical air stream, and rotor angular velocity is not remained constant per a rotor turn even if there is a steady-state auto-rotation. Hence, local angles of attack are significantly changed through the azimuths. With a view to illustrate, Fig. 3 shows distribution of the local angles of attack $\alpha_{r}$ upon a rotor disc during auto-rotation of a rotor of a GP performing nosing-up. The chart has been predicted numerically, the arrow shows a flight direction (FD).

As a rule, main rotor is designed so that aeroelasticity has got insignificant effect on its dynamical properties. As is usual, blade's deformations of bending are insignificant and fundamental mode acceptably describes motion of blades (Johnson, 1983). Nevertheless, it is required that blade flutter, divergence and resonant oscillations be studied, since it is importation for GP because its rotor angular velocity is not constant.

It is well known that aerodynamics of a main rotor is rather complicated topic (Mil' et al., 1966a; Boyd et al., 2002). Even if a rotor operates in helicopter regime and there is a level flight, aerodynamic effects are not stationary. Computational modeling of rotorcraft aerodynamics is still in its infancy and lags well behind the computational capabilities used for fixed wing (Boyd et al., 2002). Modern techniques including methods of Computational Fluid Dynamics and Discrete Vortex method do not allow one to solve all problems concerned with investigation of unsteady dynamics of a wind-milling rotor. Finally, all these methods demand only numerical computations.

The classical theory with simplified aerodynamics using empirical results of the vortex theory and experiments permits to use analytical studies (Mil' et al., 1966a). Practice shows that many special features of the dynamics can be investigated by means of

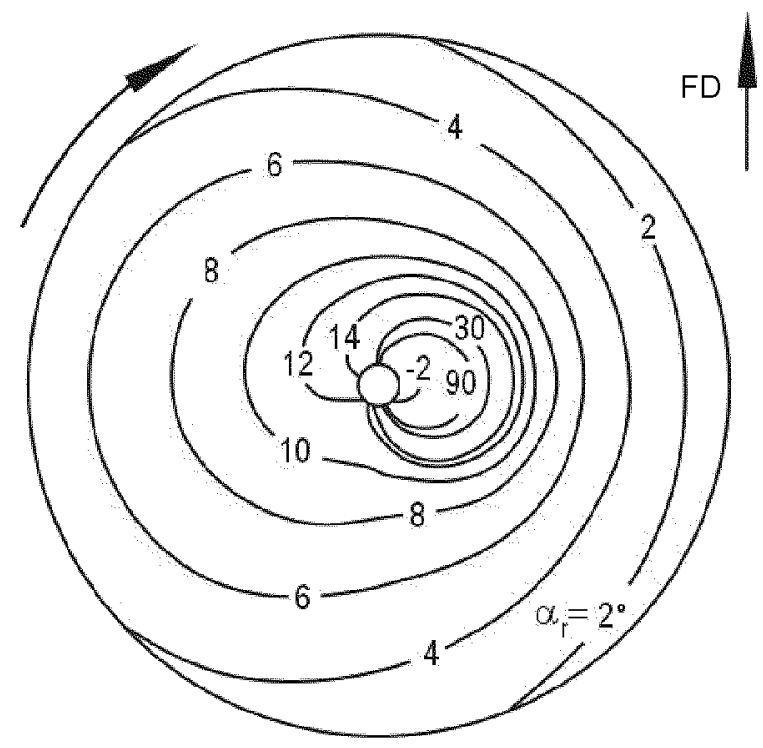

Fig. 3. Distribution of the local attack angles 


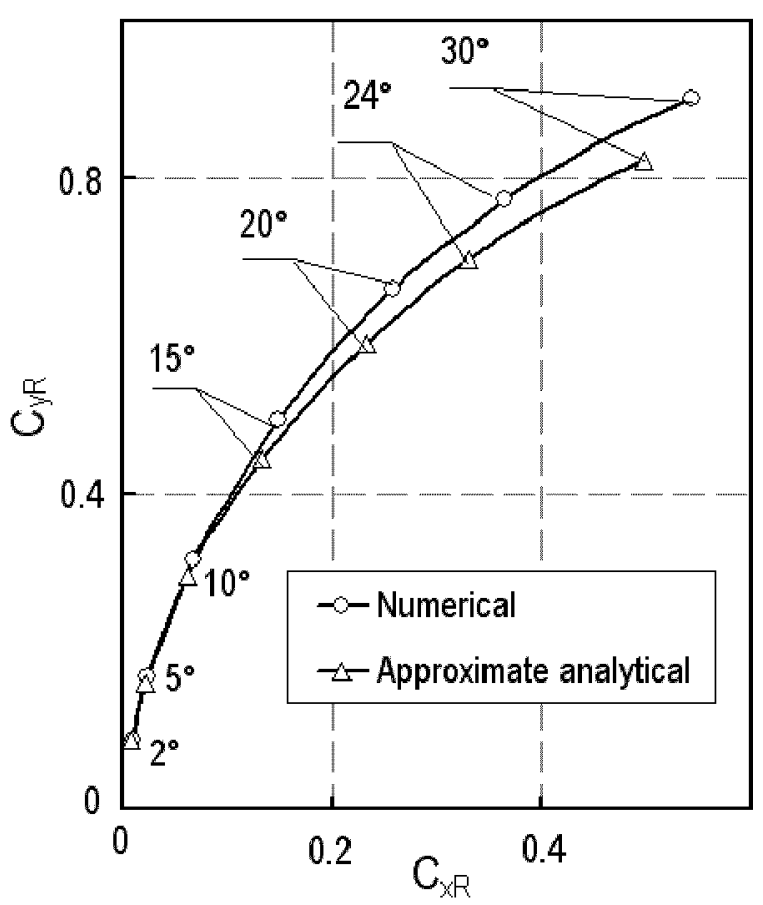

Fig. 4. Polar of the A-002 gyroplane rotor

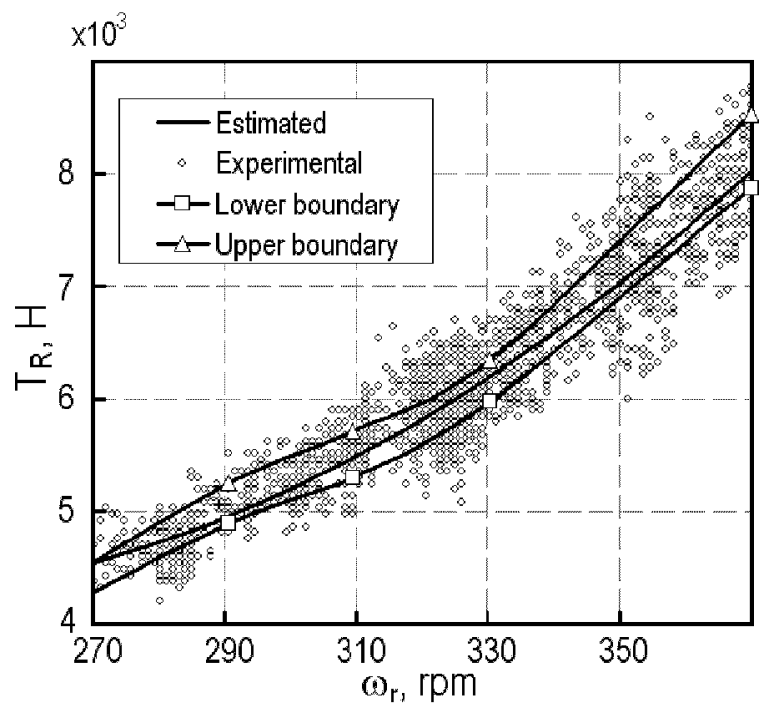

Fig. 5. A trust of the A-002 gyroplane rotor

such an approach. In the case under consideration the field of induced velocities is expressed using empirical functions. This expression permits to allow for typical features of the varying induced flow versus rotor tipspeed ratio $\mu=V_{x g} \cos \alpha_{R} /\left(\omega_{r} R\right)$, where $\alpha_{R}$ is rotor angle of attack; $\omega_{r}$ is value of the rotor angular rate averaged per one rotor turn. Taking into account redistribution of induced velocities own to curvilinear motion of an aircraft (Mil' et al., 1966a; Braverman and Vayntroob, 1988), local induced velocity is written as

$$
\begin{gathered}
v_{i}=v_{i m}\left[b+\left(p_{z} \sin \psi_{\mathrm{L}}+p_{x} \cos \psi_{\mathrm{L}}\right) \bar{r}\right] ; \\
b \equiv \sqrt{1,5 \bar{r}} \mathrm{f}_{1}(\mu)+\left(1+a \bar{r} \cos \psi_{\mathrm{aL}}\right) \mathrm{f}_{2}(\mu),
\end{gathered}
$$

where $\mathrm{f}_{1}(\mu)$ and $\mathrm{f}_{2}(\mu)$ are coefficients indicating contributions of axis-symmetrical law that is typical for low tip-speed ratios and linear law (according to Glauert hypothesis); $a$ is gradient of the linear distribution of induced velocities upon the rotor disk; $p_{z}$ and $p_{x}$ are correcting terms allowing for the redistribution because of curvilinear motion of an aircraft and rotor control angular position change; $v_{i m}$ is a mean value of induced velocities; $\psi_{\mathrm{aL}}$ is angle of an "air azimuth" of a blade; $\bar{r}=r / \mathrm{R}$, and $r$ is a bladeelement radius.

\section{ANALYTICAL SOLUTION}

While the equations of steady-state auto-rotation and flapping are solved and integral characteristics of the rotor are determined in level flight the following assumptions are accepted:

- rotor angular rate is constant and equal to the averaged value $\omega_{r}$;

- flapping angles and angles between flow and the rotor disc are low;

- blade chord and pitch are equal to values of equal untwisted untapered blade;

- influence of radial flow upon aerodynamic forces is negligible;

- tip sections of blades do not generate lift due to effect of tip losses;

- the lift coefficient of the blade section is determined by linear dependence on local attack angle;

- the profile-drag coefficient of the blade section is equal to its average value.

The flapping angle is presented by the Fourier series as a function of the azimuth angle $\psi_{\mathrm{L}}$, terminating the series after terms of first harmonic since higher terms seem to be insignificant, see Mil' et al. (1966a). Hence, flapping angle

$$
\beta_{\mathrm{L}}=a_{0}-a_{1} \cos \psi_{\mathrm{L}}-b_{1} \sin \psi_{\mathrm{L}} .
$$

An equation of steady-state auto-rotation is derived as quadratic regarding a normed axial speed of motion $x=\mathrm{V}_{x g} \sin \alpha_{\mathrm{R}} /\left(\omega_{n} \mathrm{R}\right)$. The approximate model is in rather close agreement with numerical computations and practice in the scope of its application. For example, the polar of the A-002 gyroplane rotor is presented in Fig. 4, where $\mathrm{C}_{\mathrm{yR}}$ and $\mathrm{C}_{\mathrm{xR}}$ are lift and drag coefficients, respectively. The polar was evaluated both numerically and analytically by (5) with (4) (Polyntsev, 2003a,b,c; Somov and Polyntsev, 2003, 2004). Fig. 5 shows relations of a rotor thrust on angular rates predicted and observed during windmilling of the A-002 gyroplane rotor.

\section{DYNAMICS OF FLEXIBLE ROTOR}

In order to define rotor-head loads and assess influence of blade flexibility upon rotor dynamics an equation of flexible oscillations has been derived. The blades in the plane of flapping have been modeled as beams with non-uniform distribution of parameters upon length. In the considered system with a low structural damping, allowing for dissipation of energy leads to insignificant quantitative corrections if the oscillations are far from resonance (Dondoshansky, 1965). Additionally, due to taking into account an aerodynamic damping (Mil' et al., 1966b) actual amplitudes of deflection will not be significantly changed even if the oscillations are close to resonance. The equations of the flexible oscillations during level flight, 
ignoring terms of higher order of smallness, have the form of two-dimension boundary problem

$$
\begin{aligned}
& {\left[\mathrm{EJ}_{x} y^{\prime \prime}(t, r)\right]^{\prime \prime}-\left[\mathrm{N} y^{\prime}(t, r)\right]^{\prime}+m_{r} \ddot{y}(t, r)=\mathrm{X},} \\
& {\left[\mathrm{EJ}_{y} x^{\prime \prime}(t, r)\right]^{\prime \prime}-\left[\mathrm{N} x^{\prime}(t, r)\right]^{\prime}+m_{r} \ddot{x}(t, r)=\mathrm{U} .}
\end{aligned}
$$

Here $y(t, r)$ and $x(t, r)$ are displacements of a blade element in the plane of flapping and rotation respectively with standard notations ()$^{\prime} \equiv \partial / \partial r$ and $\left(^{*}\right) \equiv d() / d t$; the functions

$$
\mathrm{N} \equiv q_{1} \dot{y}+q_{2} y+q_{3} ; \mathrm{X} \equiv q_{4} y+q_{5} ; \mathrm{U} \equiv q_{6} x+q_{7}
$$

and for denotations $S_{\alpha} \equiv \sin \alpha ; \quad C_{\alpha} \equiv \cos \alpha$;

$$
\begin{aligned}
& \beta_{\mathrm{c}} \equiv \beta_{\mathrm{L}}+a_{\mathrm{c}} ; c \equiv r+y_{0} C_{a_{\mathrm{c}}} \operatorname{tg} \beta_{\mathrm{c}} ; d \equiv \dot{\psi}_{\mathrm{L}}^{2} S_{2 \beta_{\mathrm{c}}} / 2 ; \\
& e \equiv 2 \dot{\psi}_{\mathrm{L}} \dot{x} ; f \equiv \dot{\psi}_{\mathrm{L}} \dot{\beta}_{\mathrm{L}} ; h \equiv 2 \dot{\psi}_{\mathrm{L}} \dot{y} ; g \equiv \dot{\beta}_{L}^{2}+\dot{\psi}_{L}^{2} C_{\beta_{\mathrm{c}}}^{2} ;
\end{aligned}
$$

the functions $\quad q_{1}=2 m_{r} \dot{\beta}_{\mathrm{L}} ; \quad q_{2}=m_{r}\left(\ddot{\beta}_{\mathrm{L}}-d\right)$;

$$
\begin{gathered}
q_{3}=R_{\mathrm{izL}}+m_{r}\left[g c-\left(f S_{\beta_{\mathrm{c}}}+\ddot{\psi}_{\mathrm{L}} C_{\beta_{\mathrm{c}}}\right) x-e C_{\beta_{\mathrm{c}}}\right] ; \\
q_{4}=m_{r}\left[\dot{\beta}_{\mathrm{L}}^{2}+\dot{\psi}_{\mathrm{L}}^{2} S_{\beta_{\mathrm{c}}}^{2}\right] ; \quad q_{6}=-m_{r} \dot{\psi}_{\mathrm{L}}^{2} ;
\end{gathered}
$$

$q_{5}=R_{\mathrm{iyL}}-m_{r}\left[c\left(\ddot{\beta}_{L}+d\right)+x\left(\ddot{\psi}_{\mathrm{L}} S_{\beta_{\mathrm{c}}}-f C_{\beta_{\mathrm{c}}}\right)\right]+e S_{\beta_{\mathrm{c}}}$; $q_{7}=R_{\mathrm{ixL}}+m_{r}\left[\left(h+f c-\ddot{\psi}_{\mathrm{L}} y\right) S_{\beta_{c}}+\left(\ddot{\psi}_{\mathrm{L}} c+f y\right) C_{\beta_{\mathrm{c}}}\right]$; $y_{0}$ is vertical distance between flapping hinge and cross point of blades' axes; $\mathrm{EJ}_{x}$ and $\mathrm{EJ}_{y}$ are the bladeelement rigidities; $a_{\mathrm{c}}$ is rotor coning angle; $R_{\mathrm{iyL}}$ and $R_{\mathrm{izL}}$ are components of distributed external force in the blade reference frame.

To solve the boundary problem (6)-(7) the BubnovGalerkin method has been used as the method of given forms (Morozov et al., 1995). Vector of the blade flexible displacement is presented as

$$
\mathbf{u}_{\mathrm{iL}}=\left\{\sum_{j=1}^{n} \mathrm{~g}^{(j)} \eta^{(j)} ; \quad \sum_{j=1}^{n} \mathrm{f}^{(j)} \delta^{(j)} ; 0\right\},
$$

where $\eta^{(j)}$ and $\delta^{(j)}$ are unknown amplitudes of oscillations for mode $j$ in planes of rotation and flapping, respectively; $\mathrm{g}^{(j)}$ and $\mathrm{f}^{(j)}$ are corresponding functions of natural mode shape. After transformations one can achieve the equations of forced oscillations for twoblade rotor

$$
\ddot{\delta}^{(j)}+p_{y j}^{2} \delta^{(j)}=\frac{A_{y j}}{m_{y j}} ; \ddot{\eta}^{(j)}+p_{x j}^{2} \eta^{(j)}=\frac{A_{x j}}{m_{x j}},
$$

where $p_{y j}=\left(C_{y j} / m_{y j}\right)^{1 / 2}, p_{x j}=\left(C_{x j} / m_{x j}\right)^{1 / 2}$ are free bending frequencies; generalized forces

$$
A_{y j}=\sum_{s} \int \mathrm{f}_{s}^{(j)} \mathrm{X}_{s} d r ; A_{x j}=\int\left[\mathrm{g}_{1}^{(j)} \mathrm{U}_{1}-\mathrm{g}_{2}^{(j)} \mathrm{U}_{2}\right] d r ;
$$

equivalent mass

$$
m_{y j}=\sum_{s} \int m_{r}\left[\mathrm{f}_{s}^{(j)}\right]^{2} d r ; \quad m_{x j}=\sum_{s} \int m_{r}\left[\mathrm{~g}_{s}^{(j)}\right]^{2} d r
$$

and generalized rigidities

$$
\begin{aligned}
& C_{y j}=\sum_{s} \int\left\{\mathrm{EJ}_{x}\left[\left(\mathrm{f}_{s}^{(j)}\right)^{\prime \prime}\right]^{2}+\mathrm{N}_{s}\left[\left(\mathrm{f}_{s}^{(j)}\right)^{\prime}\right]^{2}\right\} d r ; \\
& C_{x j}=\sum_{s} \int\left\{\mathrm{EJ}_{y}\left[\left(\mathrm{~g}_{s}^{(j)}\right)^{\prime \prime}\right]^{2}+\mathrm{N}_{s}\left[\left(\mathrm{~g}_{s}^{(j)}\right)^{\prime}\right]^{2}\right\} d r,
\end{aligned}
$$

and index $s$ is number of a blade.

It is seen that a rotor have damping properties. The physical sense of these properties is as follows:

- the rate of flexible displacements results in change of local attack angles so that an additional aerodynamic force damps the oscillations;
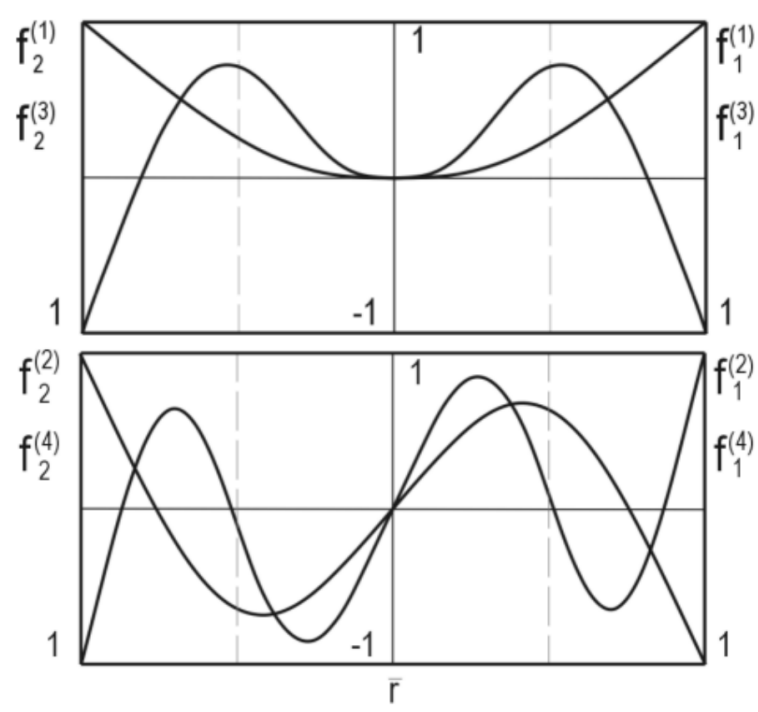

Fig. 6. The normed blades' bending modes

- the direction of external force vector is changed so that damping components are increased;

- occurrence of flapping and auto-rotation whose centrifugal forces damp the oscillations.

The equations (8) are nonlinear. This fact complicates investigation of a wind-milling rotor because flexible oscillations of the rotor effect on flapping and auto-rotation. Nekrasov (1964) recommends that such equations should be integrated numerically using given forms of free oscillations of blades. This approach allows one to take into account nonlinear dependencies that are essential to define aerodynamic forces. Advantages of this approach are: possibility to take into account blade twist and taper, to allow for aerodynamic damping more exactly and possibility to simulate flexible oscillations during regimes of unsteady auto-rotation.

If initial conditions $\delta^{(j)}\left(t_{0}\right)$ and $\dot{\delta}^{(j)}\left(t_{0}\right)$ for (8) are unknown they are set arbitrarily, for example equal to zero. For a steady motion the oscillations become steady within some revolutions (Mil' et al., 1966a; Nekrasov, 1964), i.e. the deformation amplitudes become equal to each other for the same azimuths with accuracy $10^{-3} \mathrm{R}$ (Nekrasov, 1964).

With a view to illustrate special features the rotor forced oscillations the calculations were carried out

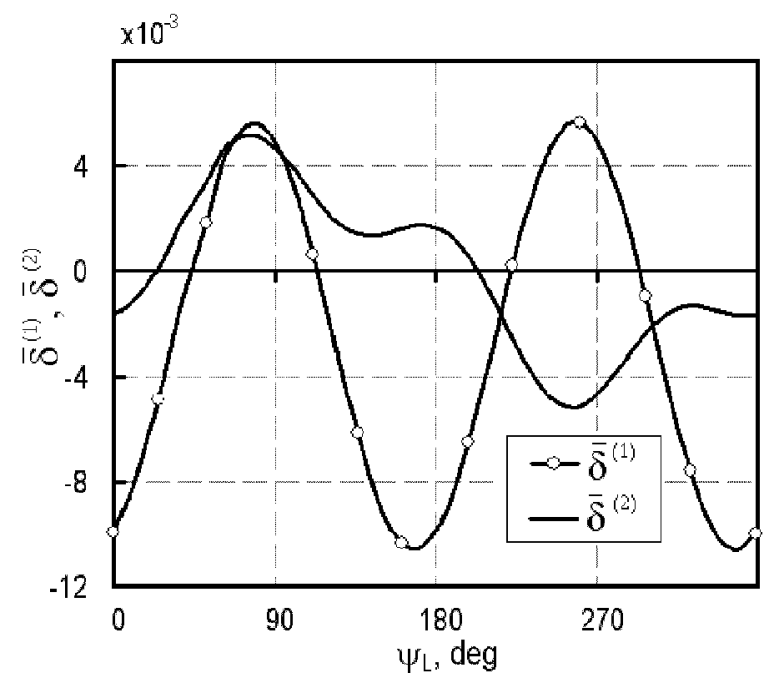

Fig. 7. The deformation coefficients upon azimuth 


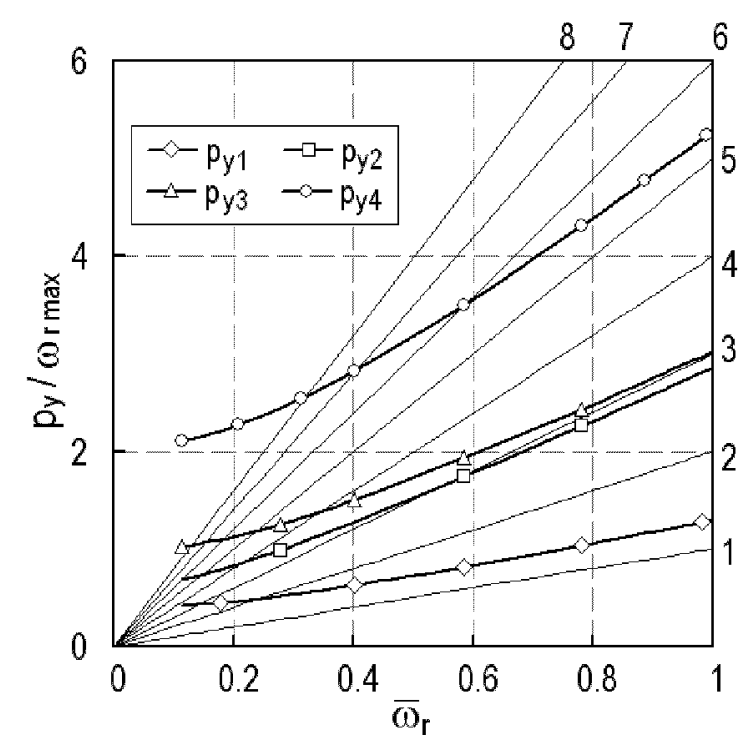

Fig. 8. The resonant diagram of the A-002 rotor

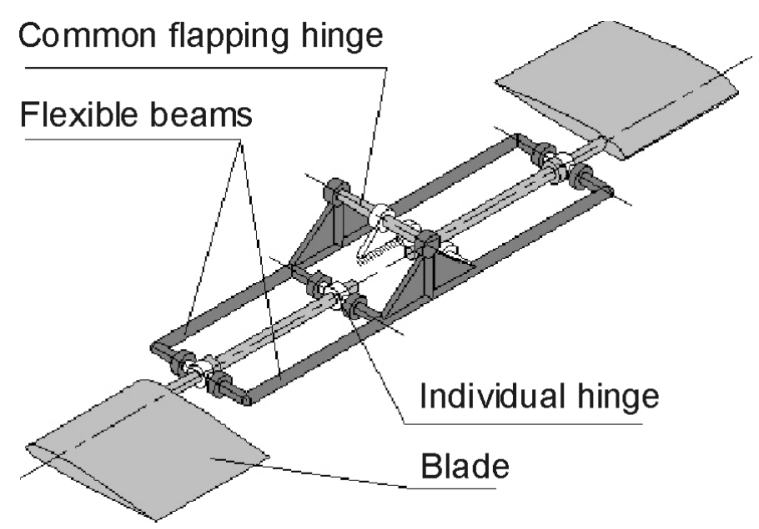

Fig. 9. A rotor-head scheme

for the A-002 gyroplane rotor. The mode shape functions have been defined by well-known software MSC NASTRAN. The special feature of the considered beam pinned in the middle is presence of symmetric and skew-symmetric forms. Fig. 6 presents 4 first shape modes in the flapping plane, excluding fundamental mode. Fig. 7 presents dependencies of so called deformation coefficients $\bar{\delta}^{(j)} \equiv \delta^{(j)} / R$ of first and second modes upon azimuth. Obviously, free frequencies of the second and third modes are close to each other - see Fig. 8, where the resonant diagram is presented. Here $\omega_{\text {rmax }}$ is maximal value of the rotor angular rate for the mode of axial auto-rotation.

Since oscillations of the first mode are stand out with frequency close to double frequency of rotor revolution the most dangerous for the considered rotor is resonant coincidence of the second-harmonic frequency with free frequency $p_{y 1}$ of first mode. This phenomenon leads to the most significant growth of oscillatory amplitudes. The same reason caused the fact that it is necessary to avoid resonant coincidence of the first-mode free bending frequency of a rotor in the rotation plane with angular rate of the rotor.

\section{DYNAMICS WITH A FLEXIBLE HUB}

An option of the A-002 gyroplane is equipped with a rather new type of a rotor-head invented by A. Tatarnikov and O. Polyntsev. Fig. 9 presents some cinematic features of the hub. The blades of the
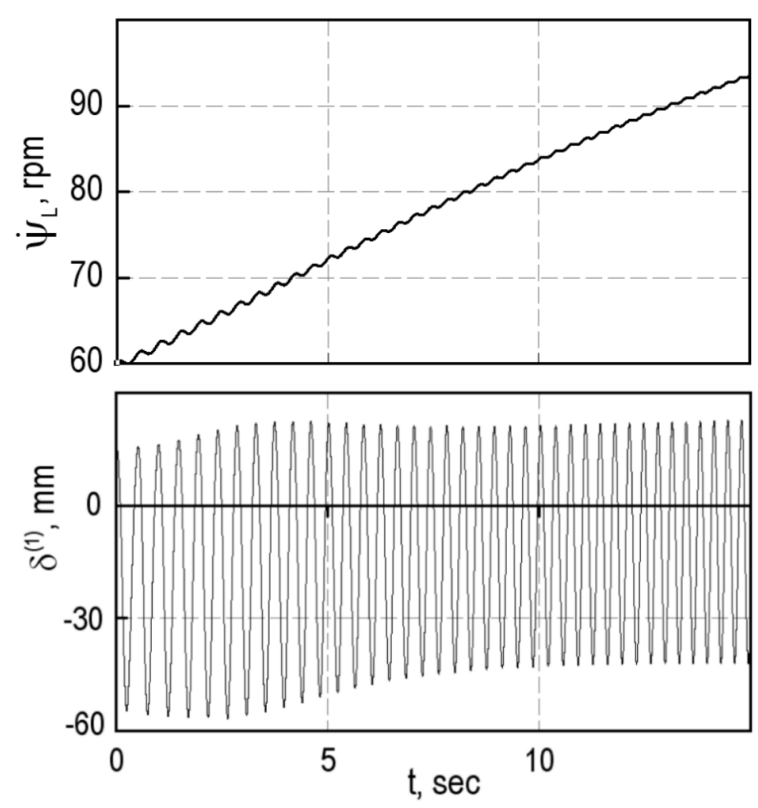

Fig. 10. The rotor rate and the blade deformations

main rotor are fully articulated and the both blades have got a common flapping hinge. Flexible beams restrict flapping and lag motions effected about axes of individual hinges. Such a construction allows GP to have a number of flight modes characterized by various ranges of loads. Elastic elements permit the hub to increase a bearing strength from the view of flapping bending moments. A centrifugal stiffening effect also occurs. It is a special interest for this GP with a collective pitch control which leads to essential change in the rotor angular rate.

Based on the elaborated mathematical models and software the controlled wind-milling rotor simulation have been carried out. As an example, Fig. 10 presents the rotor angular rate $\dot{\psi}_{\mathrm{L}}(t)$ and the deformation amplitude on first mode $\delta^{(1)}(t)$ obtained during simulation of a rotor spin-up.

\section{ROBUST CONTROL}

As well-known, a helicopter Nesterov loop performing is not a unique phenomenon. The authors considered special features of this rather sight manoeuver by a GP. Elaborated software (Kalmykov et al., 2002a,b) allows one to research the GP spatial motion at different flight modes, for instance dynamics of a main rotor pre-rotation, take-off run, take-off, climb, flying down with and without engine work, gliding, pancake, landing and landing run, the engine failure, pitchdown, other manoeuvers and unsteady regimes. There is also a possibility to define loads acting on the GP elements at different unsteady and transient modes using control stick movements.

As simulation results were stored up, an optimization of many flight modes was performed and this fact supports the improvement of aircraft dynamical features and increment of flight safety. Principal problems on a robust stabilization (Somov, 2001; Somov et al., 2003; Matrosov and Somov, 2003) of the GP flexible rotor were studied. This research was carried out associated with a ground physical experimental research and identification of the blade parameters by the GP 


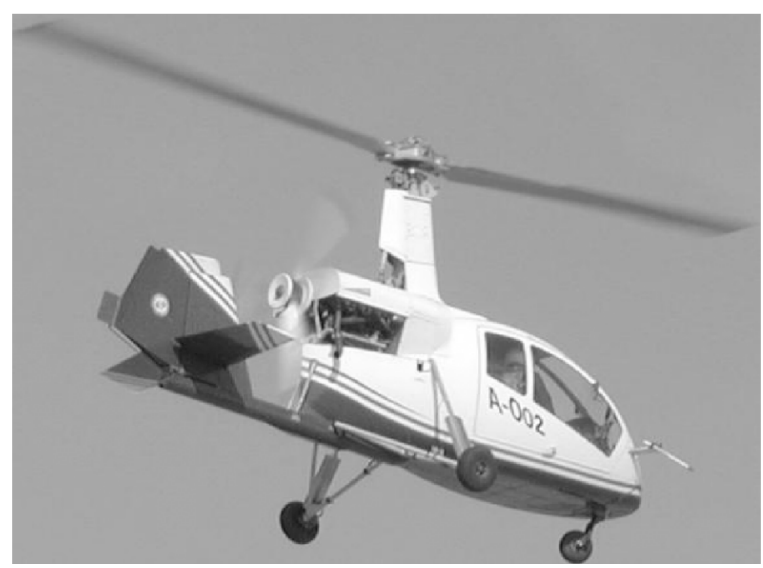

Fig. 11. The A-002 gyroplane in flight, view 1

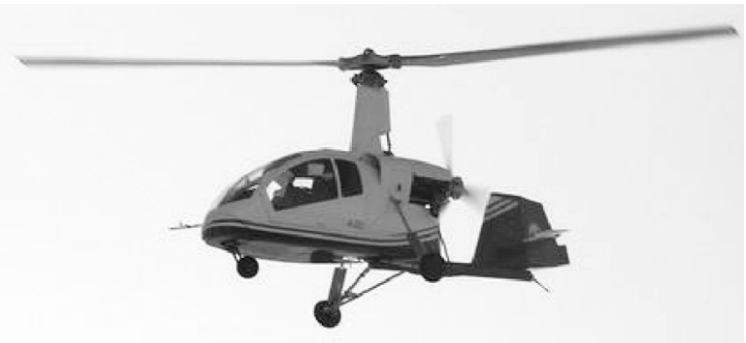

Fig. 12. The A-002 gyroplane in flight, view 2

test natural flights. In order to approve developed techniques two GPs have designed and built: twoseat aircraft in 1997 and three-seat vehicle in 2000. The GPs have been equipped with flight recorders obtaining data from the tensometer systems to define actual loads on the rotor pylon and mechanical control system. There is a rather good accordance of evaluated parameters with experimental results, for example see Fig. 5. Fruitful comparisons have been made between evaluated parameters and wind-tunnel tests performed by NACA (Polyntsev, 2003b). The results have been applied at design a main rotor of the A-002 Russian gyroplane. This aircraft was tested in flight and presented at the Moscow Aero-Space salon $M A K S$ - 2003, see Fig. 11 and Fig. 12.

\section{CONCLUSIONS}

The mathematical models describing dynamics of auto-rotation and flapping of the wind-milling rotor, were elaborated. The approximate analytical solutions has been obtained within standard parameterization. Software products allowing one to simulate rotor dynamics have been created. Major special features of forced flexible oscillations of the rotor in the flapping and rotation planes have been studied. The mathematical models have successfully been applied to design the Irkutsk A-002 gyroplane main rotor. Principal problems on a robust control and stabilization of the GP flexible rotor were studied and associated both with a ground physical experimental research and with natural flights.

\section{REFERENCES}

Boyd, D.D., R.W. Barnwell and S.A. Gorton (2002). A computational model for rotor-fuselage interactional aerodynamics. AIAA Paper (02-56), 1-10.
Braverman, A.S. and A.P. Vayntroob (1988). Helicopter dynamics. Extreme flight regimes.. Mashinostroyeniye. (Russian).

Dondoshansky, V.K. (1965). Calculations of oscillations for flexible systems by computers. Mashinostroyeniye. (Russian).

Johnson, W. (1983). Helicopter Theory. Vol. 1. Mir. Kalmykov, A.A., O.Ye. Polyntsev and Ye.I. Somov (2002a). Autogyro Nesterov loop simulation. Actual Problems of Aviation and Aerospace Systems $7(1), 56-65$.

Kalmykov, A.A., O.Ye. Polyntsev and Ye.I. Somov $(2002 b)$. Simulation of an autogyro controlled longitudinal motion. Actual Problems of Aviation and Aerospace Systems 7(1), 38-55.

Matrosov, V.M. and Ye.I. Somov (2003). Nonlinear problems of spacecraft fault tolerant control systems. Nonlinear Problems in Aviation and Aerospace, vol. 12: Advanced in Dynamics and Control. pp. 309-331. Taylor \& Francis.

Mil', M.L., A.V. Nekrasov, A.S. Braverman, L.N. Grodko and M.A. Leykand (1966a). Helicopters. Computation and Design. Vol. 1. Mashinostroyeniye. (Russian).

Mil', M.L., A.V. Nekrasov, A.S. Braverman, L.N. Grodko and M.A. Leykand (1966b). Helicopters. Computation and Design. Vol. 2. Mashinostroyeniye. (Russian).

Morozov, V.I., A.T. Ponomarev and O.V. Rysev (1995). Mathematical modeling of complex aeroflexible systems. FizMatLit. (Russian).

Nekrasov, A.V. (1964). Computation of the stress' into a helicopter's main rotor blade at a large flight speed. Proceedings of the TsAGI (898), 4071. (Russian).

Polyntsev, O.Ye. (2003a). Dynamics of a steady autorotating rotor. Actual Problems of Aviation and Aerospace Systems 8(2), 80-95.

Polyntsev, O.Ye. (2003b). Nonlinear dynamics of autorotating rotor. Actual Problems of Aviation and Aerospace Systems 8(1), 83-97.

Polyntsev, O.Ye. $(2003 c)$. Some problems of autorotating rotor dynamics. Izvestiya VUZ, ser. Aviatsionnaya Tekhnika (3), 20-24. (Russian).

Somov, Ye.I. (2001). Robust stabilization of a flexible spacecraft at partial discrete measurement and a delay in forming control. Journal of Computer and Systems Sciences International 40(2), 287-307.

Somov, Ye.I., A.G. Kozlov, V.A. Rayevsky, G.P. Anshakov and Yu.G. Antonov (2003). Nonlinear dynamic research of the spacecraft robust fault tolerant control systems. Proceedings of 15th World IFAC Congress, Barcelona 2002. Vol. G. Elsevier Science. Oxford. pp. 135-140.

Somov, Ye.I. and O.Ye. Polyntsev (2003). Nonlinear dynamics and control of a wind-milling gyroplane rotor. Proceedings of the IEEE / IUTAM International Conference "Physics and Control". Vol. 1. St.-Petersburg. pp. 152-157.

Somov, Ye.I. and O.Ye. Polyntsev (2004). Nonlinear dynamics of a gyroplane rotor. Preprints of 16th IFAC Symposium on Automatic Control in Aerospace. Vol. 1. St.-Petersburg. pp. 565-570. 